\title{
ON THE EXPLICIT FORM OF THE DENSITY OF BROWNIAN EXCURSION LOCAL TIME
}

\author{
G. HOOGHIEMSTRA
}

\begin{abstract}
Let $\underline{W}_{0}^{+}(t), 0<t<1$, denote Brownian excursion and let $\underline{l}_{0}^{+}(v), v>0$, be its local time at level $v$. Starting from a representation of the density of $\underline{l}_{0}^{+}(v)$ as a complex integral we derive an explicit form of this density, written as an infinite series involving the $n$-fold convolution of known densities. Finally the result is used as an alternative check of Knight's result on the same topic.
\end{abstract}

1. Introduction. In [2] and [4] an expression is given for the distribution of Brownian excursion local time $\underline{l}_{0}^{+}(v), v>0$. In these papers this distribution is represented as a complex integral and it is easily verified that this representation is equivalent to the double Laplace transform given in Formula (3.17) of [3] (for this verification see \$2). Although both relations seem difficult to invert (cf. [3, final part of \$3]) we have succeeded in giving an explicit form for the density of $\underline{l}_{0}^{+}(v)$, $v>0$, as an infinite series involving the $n$-fold convolution of known densities. In the final part of this note it is shown that our result agrees with that of Knight (cf. [5]).

To read this note no knowledge of complex integration other than the inversion theorem for Laplace transforms is necessary.

2. The explicit form. Denote by $S$ the path (straight line) from $a-i \infty$ to $a+i \infty, a>0$. Then according to [2, Formula (5.9)] or [4, Formula VI (3.36)] for $v>0$ and $y \geq 0$,

$$
\begin{aligned}
P\left\{\underline{l}_{0}^{+}(v)\right. & \leqslant y\} \\
& =1-\frac{1}{2 i \sqrt{2 \pi}} \int_{S} \sqrt{z} e^{z / 2} \frac{e^{-v \sqrt{z}}}{\sinh \{v \sqrt{z}\}} \exp \left\{-\frac{y}{2} \frac{\sqrt{z} e^{v \sqrt{z}}}{\sinh \{v \sqrt{z}\}}\right\} d z,
\end{aligned}
$$

where $\underline{l}_{0}^{+}(v)$ is the local time at level $v>0$ of Brownian excursion. To obtain the equivalence of (2.1) with Formula (3.17) of [3], observe that if $\underline{z}(\cdot, t)$ is the local time of the excursion straddling $t$, and $\underline{l}(t)$ its length, then

$$
\underline{z}(v, t) \stackrel{d}{=}(\underline{l}(t))^{1 / 2} \underline{l}_{0}^{+}\left(v(\underline{l}(t))^{-1 / 2}\right),
$$

with $\underline{l}_{0}^{+}(v)$ and $\underline{l}(t)$ independent.

Since the distribution of $l(t)$ is known (cf. [1, (4.4)]), it is easy to obtain Formula (3.17) of [3] from Relation (2.1). W. Vervaat (private communication) has pointed

Received by the editors February 27, 1981.

1980 Mathematics Subject Classification. Primary 60J55, 60 J65.

(C) 1982 American Mathematical Society 0002-9939/82/0000-0029/\$02.00 
out that the converse statement is also true, because it follows from Relation (2.2) and Formula (3.17) of [3] that for $\mu, \rho \geqslant 0$,

$$
\frac{1}{\sqrt{2 \pi}} \int_{0}^{\infty} \frac{e^{-u x}}{x^{3 / 2}}\left(1-E \exp \left(-\rho \sqrt{x} \underline{l}_{0}^{+}\left(x^{-1 / 2}\right)\right)\right) d x=\frac{2 \rho \sqrt{2 u} e^{-2 \sqrt{2 u}}}{\sqrt{2 u}+\rho\left(1-e^{-2 \sqrt{2 u}}\right)} .
$$

The latter relation uniquely determines Relation (2.1).

We shall derive an explicit expression for the density of $\underline{l}_{0}^{+}(v)$. Observe from (2.1) that the density of $\underline{l}_{0}^{+}$at level $v$ is given for $y>0$ by

$$
\frac{1}{4 i \sqrt{2 \pi}} \int_{S} e^{z / 2} \frac{z}{\sinh ^{2}\{v \sqrt{z}\}} \exp \left\{-\frac{y}{2} \frac{\sqrt{z} e^{v \sqrt{z}}}{\sinh \{v \sqrt{z}\}}\right\} d z .
$$

Now use the fact that

$$
\frac{\sqrt{z} e^{v \sqrt{z}}}{2 \sinh \{v \sqrt{z}\}}=\sqrt{z}+\frac{\sqrt{z} e^{-v \sqrt{z}}}{2 \sinh \{v \sqrt{z}\}}
$$

and the series expansion of $\exp (\cdot)$ to obtain, for $y, v>0$,

$$
\begin{aligned}
& \frac{d}{d y} P\left\{\underline{l}_{0}^{+}(v) \leqslant y\right\} \\
& \quad=\left(4 i v^{2} \sqrt{2 \pi}\right)^{-1} \sum_{n=0}^{\infty} \frac{(-y / 2 v)^{n}}{n !} \int_{S} e^{z / 2} \frac{v^{2} z}{\sinh ^{2}\{v \sqrt{z}\}}\left[\frac{v \sqrt{z} e^{-v \sqrt{z}}}{\sinh \{v \sqrt{z}\}}\right]^{n} e^{-y \sqrt{z}} d z .
\end{aligned}
$$

We define $x:=y / v, \lambda:=v^{2} z / \pi^{2}$. From this substitution it follows that the r.h.s. of (2.4) equals

$$
\frac{\pi^{2}}{4 i v^{4} \sqrt{2 \pi}} \sum_{n=0}^{\infty} \frac{(-x / 2)^{n}}{n !} \int_{S} e^{\lambda\left(\pi^{2} / 2 v^{2}\right)} \frac{4 \pi^{2} \lambda e^{-2 \pi \sqrt{\lambda}}}{\left(1-e^{-2 \pi \sqrt{\lambda}}\right)^{2}}\left[\frac{2 \pi \sqrt{\lambda} e^{-2 \pi \sqrt{\lambda}}}{1-e^{-2 \pi \sqrt{\lambda}}}\right]^{n} e^{-x \pi \sqrt{\lambda}} d \lambda .
$$

It is known from [1, p. 168] that

$$
\begin{gathered}
\int_{0}^{\infty} e^{-\lambda s} d F_{1}(s)=\frac{2 \pi \sqrt{\lambda} e^{-\pi \sqrt{\lambda}}}{\left(1-e^{-2 \pi \sqrt{\lambda}}\right)}, \\
\int_{0}^{\infty} e^{-\lambda s} d F_{2}(s)=\frac{4 \pi^{2} \lambda e^{-2 \pi \sqrt{\lambda}}}{\left(1-e^{-2 \pi \sqrt{\lambda}}\right)^{2}},
\end{gathered}
$$

where $F_{1}$ and $F_{2}$ are proper distribution functions $\left(F_{1}\left(2 s^{2}\right), F_{2}\left(2 s^{2}\right)\right.$ is the distribution function of the maximum of Brownian meander, the maximum of Brownian excursion, respectively) defined by

$$
\begin{aligned}
& F_{1}(s):=\sum_{n=-\infty}^{\infty}(-1)^{n} e^{-n^{2} s}, \quad s>0, \\
& F_{2}(s):=1+2 \sum_{n=1}^{\infty} e^{-n^{2} s}\left(1-2 n^{2} s\right), \quad s>0 .
\end{aligned}
$$


Note that if $f_{i}$ is the density of $F_{i}$, then $f_{2}=f_{1}^{2 *}$ (cf. [1] or [5]). Further it is well known that for $k>0$

$$
\int_{0}^{\infty} e^{-\lambda s} \frac{k}{2 \sqrt{\pi s^{3}}} e^{-k^{2} / 4 s} d s=e^{-k \sqrt{\lambda}}
$$

Hence, it follows from (2.4)-(2.10) and the Laplace inversion theorem that for $y, v>0$

$$
\frac{d}{d y} P\left\{\underline{l}_{0}^{+}(v) \leqslant y\right\}=2^{-3 / 2} \pi^{5 / 2} v^{-4} \sum_{n=0}^{\infty} \frac{(-y)^{n}}{n ! 2^{n} v^{n}} g_{x} * f_{2} *\left\{g_{1} * f_{1}\right\}^{n *}\left(\frac{\pi^{2}}{2 v^{2}}\right),
$$

where the densities $f_{i}$ and $g_{x}$ on $(0, \infty)$ are defined by

$$
f_{i}(u)=F_{i}^{\prime}(u), \quad i=1,2, \quad g_{x}(u)=\frac{x \sqrt{\pi}}{2 u^{3 / 2}} e^{-x^{2} \pi^{2} / 4 u}, \quad x>0 .
$$

Recently F. B. Knight obtained in an entirely different way an explicit form for the density of $\underline{l}_{0}^{+}(v)$. Knight's result (cf. [5]) states that, for $y, v>0$,

$$
\frac{d}{d y} P\left\{\underline{l}_{0}^{+}(v) \leqslant y\right\}=2^{-1 / 2} \pi^{5 / 2} v^{-3} \int_{0}^{1} f_{2}\left(\frac{\pi^{2}}{2 v^{2}}(1-t)\right) f(t, y) d t,{ }^{1}
$$

where the density $f_{2}$ is defined above and

$$
f(t, y):=-\frac{(2 \pi t)^{-1 / 2}}{2 v} \sum_{n=0}^{\infty} \frac{1}{n !} \frac{d^{n-1}}{d y^{n-1}}\left\{y^{n} \frac{d^{2}}{d y^{2}} e^{-2 v^{2}(y+n)^{2} / t}\right\} .
$$

A direct verification (without Laplace transforms) of the equivalence of (2.11) and (2.12) seems too difficult. For this reason we follow the final remark of Knight in [5], which states that the r.h.s. of (2.12) is a convolution and that passing to Laplace transforms might give a simplification of (2.12). Knight states that the Laplace transform then obtained seems too difficult for inversion but in fact it turns out that this Laplace transform is equivalent with (2.3); consequently (2.11) and (2.12) give the same expression and the desired simplification of (2.12) is relation (2.11). We shall prove this in the following.

Observe that the r.h.s. of $(2.12)$ can be written as

$$
2^{1 / 2} \pi^{1 / 2} v^{-1} \int_{0}^{\pi^{2} / 2 v^{2}} f_{2}\left(\frac{\pi^{2}}{2 v^{2}}-u\right) f\left(\frac{2 v^{2}}{\pi^{2}} u, y\right) d u \text {. }
$$

The integral is a convolution integral. The joint Laplace transform of $f(t, y)$ in the variables $\lambda$ and $\beta$ is given by (cf. [5, Lemma 2.2]),

$$
(2 v)^{-1} \frac{1-e^{-2 v \sqrt{2 \lambda}}}{\sqrt{2 \lambda}+\beta\left(1-e^{-2 v \sqrt{2 \lambda}}\right)} .
$$

\footnotetext{
${ }^{1}$ As remarked by the author himself, the density in Theorem 2.3 of [5] is a factor four too large due to an oversight in Theorem 1.1 of [5] (cf. [6]).
} 
The Laplace transform in $\beta$ is a simple Laplace transform of the negative exponential distribution, hence

$$
\int_{0}^{\infty} e^{-\lambda t} f(t, y) d t=(2 v)^{-1} \exp \left\{-\frac{y}{2} \frac{\sqrt{2 \lambda} e^{v \sqrt{2 \lambda}}}{\sinh \{v \sqrt{2 \lambda}\}}\right\} .
$$

The relations (2.7) and (2.15) imply that

$$
\begin{aligned}
f_{2}(t) & =(2 \pi i)^{-1} \int_{S} e^{\lambda t} \frac{\pi^{2} \lambda}{\sinh ^{2}\{\pi \sqrt{\lambda}\}} d \lambda, \\
f(t, y) & =(2 \pi i)^{-1} \int_{S} e^{\lambda t}(2 v)^{-1} \exp \left\{-\frac{y}{2} \frac{\sqrt{2 \lambda} e^{v \sqrt{2 \lambda}}}{\sinh \{v \sqrt{2 \lambda}\}}\right\} d \lambda .
\end{aligned}
$$

It follows from (2.14)-(2.17) and the substitution $z=\pi^{2} \lambda / v^{2}$ that the density given by Knight's formula is equal to

$$
(4 i \sqrt{2 \pi})^{-1} \int_{S} e^{z / 2} \frac{z}{\sinh ^{2}\{v \sqrt{z}\}} \exp \left\{-\frac{y}{2} \frac{\sqrt{z} e^{v \sqrt{z}}}{\sinh \{v \sqrt{z}\}}\right\} d z .
$$

Indeed the latter expression is equal to the one in Formula (2.3).

REMARK. Integration of (2.3) over $y$ from 0 to $\infty$ leads to

$$
(i \sqrt{2 \pi})^{-1} \int_{S} e^{z / 2} \frac{\sqrt{z} e^{-2 v \sqrt{z}}}{1-e^{-2 v \sqrt{z}}} d z .
$$

This expression has to be equal to $1-F_{2}\left(2 v^{2}\right)$, since the density of the local time has an atom of magnitude $F_{2}\left(2 v^{2}\right)$ at $0\left(F_{2}\left(2 v^{2}\right)\right.$ is the probability that the maximum of Brownian excursion is smaller than $v$ ). Indeed this equality is easily verified by expanding the numerator of the integrand in (2.19) in powers of $\exp \{-2 v \sqrt{z}\}$ and by using Relation (2.10).

Because it is also readily verified from (2.6) and (2.10) that Expression (2.19) equals $\left(\pi^{2} \sqrt{2 \pi} / 2 v^{3}\right)\left(f_{1} * g_{1}\right)\left(\pi^{2} / 2 v^{2}\right)$, we have as by-product the relation

$$
\left(f_{1} * g_{1}\right)\left(\frac{\pi^{2}}{2 v^{2}}\right)=\frac{2 v^{3}}{\pi^{2} \sqrt{2 \pi}}\left(1-F_{2}\left(2 v^{2}\right)\right) \text {. }
$$

Relation (2.20) can be used to give an alternative form of (2.11).

\section{REFERENCES}

1. K. L. Chung, Excursions in Brownian motion, Ark. Mat. 14 (1976), 155-177.

2. J. W. Cohen and G. Hooghiemstra, On the occupation time of Brownian excursion, Preprint 148, Univ. of Utrecht; Math. Oper. Res. (to appear).

3. R. K. Getoor and M. J. Sharpe, Excursions of Brownian motion and Bessel processes, Z. Wahrsch. Verw. Gebiete 47 (1979), 83-106.

4. G. Hooghiemstra, Brownian excursion and limit theorems for the $M / G / 1$ queue, $\mathrm{Ph} . \mathrm{D}$. thesis, Univ. of Utrecht, 1979.

5. F. B. Knight, On the excursion process of Brownian motion, Trans. Amer. Math. Soc. 258 (1980), 77-86.

6. , Zbl. Math. 426 (1980), abstract 60073.

Department of Mathematics, University of Utrecht, Budapestlaan 6, 3508 TA Utrecht, The NETHERLANDS 